\title{
Clásicos de la teoría de la administración pública
}

\section{Omar Guerrero*}

\section{Resumen}

El propósito de este trabajo es estudiar las contribuciones que en el campo de la administración pública realizaron cuatro pensadores del siglo $X \mid X$ y comienzos del siglo $X X$. Los resultados revelan que Florentino González (México) introduce la ciencia de la Administración en América y se detiene en la administración local con lo cual marca una diferencia con las fuentes europeas que le sirvieron de base. Alejandro Vivien (Francia) rompe desde la perspectiva de las instituciones políticas con el monopolio del derecho administrativo en Francia. Adolfo Posada (España) si bien no aporta nuevos conocimientos, contribuye a la recuperación de la ciencia administrativa en España y Europa. Finalmente William Willoughby Estados Unidos) tiene el mérito de haber introducido el concepto de Ciencia de la Administración Pública para identificar la disciplina, haciendo aportes epistemológicos a la discusión de la materia en los Estados Unidos. Se concluye que se trata de cuatro pensadores cuyas contribuciones es necesario estudiar en el análisis del pensamiento sobre la administración pública.

Palabras claves: Administración püblica, Florentino González, Alejandro Vivien, Adolfo Posada, William Willoughby.

\section{Clasical theorists of public administration}

\section{Abstract}

The purpose of this paper is to study the contributions of four theorists in the area of public administration from the 19 th century and the beginning of the 20 th century. The

\section{Recibido: 20-01-97 Aceptado: 03-03-97}

- Licenciado en Ciencias Politicas (UNAM-México). Doctor en Administración Pública (UNAM). Profesor e investigador de Teoría de la Administración Pública en la Facultad de Ciencias Políticas y Sociales de la UNAM. Autor de numerosos trabajos publicados en fibros y artículos científicos. 
results reveal that Florentino Gonzalez (Mexico) introduced the science of administration in America and focused on local administration which was a major change from the focus of the European thinkers who served as his mentors. Alejandro Vivien (France) broke away from the monopolistic emphasis of the French school on administrative law in his analysis of political institutions. Adolfo Posada (Spain) although not offering new perspectives, contributed to the recovery of the administrative sciences in Spain and Europe. Finally, William Willoughby (United States) has the honor of having introduced the concept of the Science of Public Administration in order to identify the discipline, and made significant epistemological contributions in the discussion of this field in the United States. The conclusion is that these are four theorists whose works should be studied in the analysis of the theory of public administration.

Key words: Public administration, Florentino González, Alejandro Vivien, Adolfo Posada, Willam Willoughby.

\section{Introducción}

En 1986 culminé un trabajo que me habia ocupado por cinco años: trazar el desarrollo de la teoría de la administración pública, de su origen hasta nuestros días. En varias obras quedó plasmado el saldo de estos trabajos, que abarcaron publicaciones en la Revista de Administración Pública de México, así como en libros y artículos. En estos trabajos se realizó un esfuerzo de construcción de la Teoría de la Administración Pública a partir de lo que, en el siglo XVIII, se conocía como Ciencia de la Policía y que era la disciplina central dentro de las Ciencias Camerales, tal como eran cultivadas en los principados alemanes y profesadas en sus universidades. Después seguimos su trazo histórico hacia el siglo $\mathrm{XIX}$, para arribar finalmente al siglo actual, no sin antes haber asumido el nombre de Ciencia de la Administración.

Particularmente se puso atención en resaltar el papel de aquellos pensadores más destacados, pues a partir de sus aportaciones pudimos observar la formación de corrientes de pensamiento afines que formaban varias generaciones de cultivadores de la Ciencia de la Administración. Especial tratamiento merecieron Juan Enrique von Justi, Carlos Juan Bonnin, Lorenzo von Stein y José Posada de Herrera, además del mexicano Luis de la Rosa, quienes estuvieron activos durante los siglos XVIII y XIX, y fungen como columnas sólidas de la Ciencia de la Administración. No se soslayaron otras contribuciones, tales como las de Woodrow Wilson, Carlos Ferraris, Alejandro Oliván y otros pensadores no menos conspicuos.

A pesar de que los mencionados trabajos recogían contribuciones harto significativas, y comprendían a los pensadores que más habian significado en la construcción y desarrollo de la Ciencia de la Administración, habian quedado fuera algunos cultivadores ciertamente importantes debido a la imposibilidad de acceder a sus trabajos. Ellos son Florentino González, Alejandro Vivien, Adolfo Posada y William Willoughby. Este artículo tiene por objeto subsanar este deficitario temático, inexcusable para un ofrecimiento cabal del panorama de la Teoria de la 
Administración Pública, universalmente considerada. Sin ellos, la galería de clásicos del pensamiento administrativo estaba incompleta.

\section{Florentino González o La Introducción de la Ciencia Administrativa en Iberoamérica}

En 1840, cuando la Ciencia de la Administración se hallaba casi extinta en Francia, en tanto que en Alemania aún se seguía cultivando la antigua Ciencia de la Policía, sólo España dedicaba su esfuerzo al estudio de la administración pública. En esos tiempos inició su actividad un personaje nacido en Nueva Granada, hoy Colombia, a quien se le había solicitado en el Colegio de San Bartolomé, en Bogotá, que impartiera un curso de Ciencia Administrativa.

Su trascendental tarea estuvo apoyada en Bonnin, cuyos Principios de Administración Pública habian tenido varias ediciones y fue traducido al español $y$ al italiano (Bonnin, 1812) ${ }^{1}$. Sin embargo, como republicano convencido y amante del Gobierno local, observaba en la obra del pensador francés a un régimen centralizado que no tenía paralelo en su patria. Por lo tanto, fue la aparición de la Democracia en América de Alejandro de Tocquevilie lo que complementó sus fuentes básicas para el estudio de la administración local -además del uso del digesto del señor Gordon, sobre la administración pública británica- y así, para nutrir sus lecciones, escribió los Elementos de Ciencia Administrativa, dentro de los cuales había formado un bosquejo de lo que llamaba un Sistema de Administración Pública para un Estado Repubicano (González, 1840) ${ }^{2}$.

Con la preparación de esta obra monumental se inaugura en América toda, y especialmente en los paises de habla hispana, el cultivo de la Ciencia de la Administración.

González dedicó los dos años previos a la publiçación del libro, a profesar cátedra y prepararlo, además de divulgar su plan de trabajo (en el Periódico, Bandera Nacional) y alcanzar la atención de los miembros de la Cámara de Representantes para la redacción de un nuevo

Tres volúmenes. La edición original es de 1808, seguida por otra de 1809 y la referida, de 1812, la más consultada. En 1829 apareció un Compendio de los Principios, versión de la cual se tradujo al español en 1834, en tanto que en 1824 la obra íntegra se había trasladado al italiano.

2 En agosto de 1973 adquiri un valiosísimo libro titulado Historia de la Administración Española e Hispanoamericana, cuyo autor es el erudito investigador Juan Beneyto (Madrid, Aguitar. 1958). En el Apéndice a la Introducción de la obra, se consignaba la existencia del de González. Después de dos breves visitas a Santafé de Bogotá, que no me facilitaron la búsqueda del libro, tuvo la oportunidad de regresar a la Ciudad y dedicar tiempo a su localización. Y en efecto, en breve tiempo di con el libro en la Biblioteca Nacional y gracias a las gentilezas de su directora, así como al apoyo del Dr. Julio Roballo, director general de la Escuela Superior de Administración Pública, tuve acceso a una copia de la obra, aquí utilizada. Recientemente tuve el placer de preparar el Estudio Introductorio de los Elementos de Ciencia Administrativa, publicados por la Escuela Superior de Administración Pública de Colombia. 1994. pp. 7-37. 
Código Administrativo fundado en los principios de la descentralización. Por fin, luego de sus experiencias docentes e investigativas, Florentino González podía decir con toda modestia que, con yerros propiamente humanos, habia escrito "un libro que trata de una ciencia desconocida en América, y una ciencia que nos importa mucho cultivar, si queremos ser algún día felices, este libro es de una gran utilidad, aunque no de más que bases para discernir. Él despierta ciertas ideas, que desarrollándose por talentos superiores, tendrán con el tiempo una trascendencia inmensa sobre la dicha social". Tratándose de una ciencia nueva en América, su objeto es poner a los jóvenes en el camino para arribar a la organización administrativa más perfecta que se tiene, más que disertar para los estadistas que por principio tienen sabiduria en los contenidos de la obra.

Uno de los méritos más grandes de Florentino González, consiste en haber introducido la Ciencia de la Administración a nuestro Continente, y por consiguiente, a su moderno fundador: Carlos Juan Bonnin, a pesar de desilusión que le cause el que los Principios se refieran a un régimen centralizado. Así, para dar pauta al Sistema de Administración Pública que ha elaborado, comienza ofreciéndonos una definición del objeto de su libro, la Ciencia Administrativa, de la que dice que "es el conocimiento de los principios, en virtud de los cuales debe arreglarse la acción de las autoridades a quienes se encargue el manejo de los intereses y negocios sociales, que tengan el carácter de públicos".
Ahora bien, en cuanto a dichos intereses, ellos se refieren a los que son inherentes a la condición del individuo como miembro de la sociedad, y por lo tanto a la sociedad en sí, que contrastan con aquellos otros de tipo privado que se refieren a los que el individuo cuida por motivos de su propia utilidad. En contraste a éstos intereses privados, tales como el ejercicio del pensamiento y de la industria, y la profesión de un culto religioso, los intereses sociales se refieren a las poblaciones, el usufructo de los productos naturales, la seguridad de los habitantes, la educación pública, el matrimonio, los decesos y las contribuciones, y que por naturaleza tienen un carácter público.

Una vez que se han identificado los intereses sociales, González explica que "la administración pública es la acción de las autoridades sobre los intereses y negocios sociales, que tengan el carácter público, ejercida conforme a las reglas que se hayan establecido para manejarlos". Esta idea, que se refiere a la acción, el autor la extiende a los funcionarios mismos, de modo que lo que llama una buena administración, tal como lo subraya, se refiere tanto a los resultados ventajosos que se obtienen, como a los funcionarios que la ejercitan. De acuerdo a su proyecto, Florentino González distingue a la administración pública nacional y a la administración municipal, entendiendo a la primera como la acción de la autoridad pública sobre los intereses y negocios sociales relativos a la nación, y a la segunda la acción de la autoridad pública sobre los intereses y negocios referentes a las secciones de la nación. 
Finalmente unas palabras más sobre el temario y capitulado de la obra, integrada por libros como se acostumbraba antaño. El primero comprende un capítulo sobre las ideas generales de administración, y sigue con el tratamiento de los medios pasivos para facilitar la administración pública. El capítulo III se refiere a los medios activos, en tanto que el IV al movimiento administrativo. El libro segundo está integrado 14 capítulos, todos ellos referentes a la administración nacional, y tratase en su interior la organización de la administración pública y sus diversos funcionarios. Finalmente, el tercer libro, plasmado en todo el tomo II, estudia a la administración municipal, su organización y sus agentes.

La labor de Florentino González, padre de la Ciencia de la Administración en América, tuvo la emulación en México de Luis de la Rosa cuya obra La Administración Pública de México y Medios de Mejorarla apareció en 1853, en tanto que otro González -Antonio González Saravia-, sacaba de imprenta su libro La Administración Pública en 1888.

\section{Alejandro Vivien o El Renacimiento de la Ciencia Administrativa en Francia}

Hacia 1845 la Ciencia de la Administración ya había sido introducida en
América, mientras que su estudio en Francia había languidecido. Bonnin no había dejado discípulos en su propio país, era más conocido y reconocido en España e Hispanoamérica, y no faltaba mucho para que Lorenz von Stein lo adoptara como maestro y renovara en Alemania a la antigua Ciencia de la Policía, para convertirla en la Teoria de la Administración. ${ }^{3}$

Fue entonces que aparecieron los Estudios Administrativos de Alejandro Vivien $(1974)^{4}$, cuya publicación vino a contrastar, y desafiar, el dominio monopolístico que ejercía el derecho administrativo en Francia.

Los Estudios de Vivien constituyen un tratamiento cabal de la administración pública francesa de sus días. El primer volumen sitúa a la administración dentro de las instituciones politicas, y desenvuelve un tratamiento muy preciso de cada uno de los órganos de aquella, tales como los Consejos, tribunales y otros más, así como de los funcionarios y los procedimientos y formas de la administración. Finalmente, el segundo volumen está dedicado a la administración local, la policía, los cultos y los espectáculos públicos.

La importancia de Vivien consiste en que su libro es una alternativa a los tratados de derecho administrativo, pero también a su idea de la administración pública en función de las instituciones

3 La obra de Lorenz von Stein se titula: Die Verwaltungs-Lehre (Sttutgart, Berlang der F.G. Gottasfchen Buchlandung. 1861. Ocho volúmenes). Nosotros consulatamos la versión italiana: Stein, Lorenz von. La Scienza della Pubblica Amministrazione. Torino, Unione Tipografico-Editrice. 1897.

4 La edición original es de 1845 , seguida por otra de 1852 y una más de 1859 , de la cual se obtuvo la edición facsímil aqui utilizada. 
políticas. Explica que, en su infancia, las sociedades solo conocían una confusión de poderes públicos y no fue sino hasta que se desarrolló, y se hizo más compleja cuando surgió una necesaria división entre ellos. Pero este mismo proceso provocó que, en su seno, el Poder Ejecutivo propiciara la formación de dos ramas: la política y la administración.

Define a la política como "la dirección de moral de los intereses generales de la nación", en tanto que la administración "consiste principalmente en provisión de los servicios públicos". Así, los regímenes modernos han desarrollado la doble división de poderes que distingue los Poderes Legislativo, Ejecutivo y Judicial, al mismo tiempo que la política también se ha extendido a las Asambleas legislativas, a las cuales competen, asimismo, las grandes cuestiones nacionales. A ellas toca también formar las leyes, intervenir en las relaciones exteriores, y les preocupa la seguridad y la salud del pueblo.

Por su parte, la administración aplica lo que el legislador dispone. Este declara el derecho, aquella lo ejecuta. El legislador tiene la elevada función de ejercitar la autoridad más plena, en tanto que "la administración no tiene sino un papel subordinado, pero ella abraza los objetos más numerosos, traduce los hechos, toca las realidades políticas". Aunque secundaria, la administración atiende los grandes intereses de la nación y es un resorte considerable y necesario de la organización política.

Por otra parte, la administración tiene una relación estrecha con el poder político, pues éste "es la cabeza y la ad- ministración los brazos". Al poder político toca la iniciativa, la dirección y el consejo, da a la administración su espíritu general, su pensamiento $y$, si se puede decirlo así, su bandera. Toca a la administración la acción, la ejecución de las leyes y el ejercicio material y práctico de los poderes confiados al Gobiemo. Vivien razona que la administración arma las defensas, organiza a las obras públicas y fomenta el comercio, materializando asi las leyes.

Vivien tampoco dejó discípulos, su obra principal no se perpetuó a través de una corriente de pensamiento, pues el derecho administrativo permaneció dominante, y absorbente, como ocurrió también en España en la segunda mitad del siglo XIX.

\section{Adolfo Posada o La Reivindicación de la Ciencia Administrativa Española}

Hacia mucho tiempo que se habia dejado de cultivar la ciencia de la administración en España, y estaban casi olvidados Silvera, Oliván y Posada de Herrera (Silvera, 1839; Oliván, 1843; Posada de Herrera, 1843), cuando Adolfo Posada produjo su libro La Administración Política y la Administración Social. Eran los últimos años del siglo XIX, principiaba en breve el siglo $\mathrm{XX}$.

Posada representa la más impresionante recuperación de la ciencia administrativa, tanto en España como en Europa, por efecto de la divulgación de las obras de von Stein. Como los pensadores italianos de su época, a los que cita fre- 
cuentemente, Posada es un discípulo directo de Stein.

Parte de la actividad del Estado, como von Stein, en el sentido que su objeto es una necesidad que satisface, a saber, la conservación y perfecciona. miento de la sociedad. Tratándose de administración, toda su actividad se encamina a este propósito, y la amplitud de la esfera del Estado, y su grado de desarroIlo, determinan su organización y el número de los ministerios que atienden la variedad de funciones especificas que se desprenden de esta actividad. En la medida en que el Estado se orienta a un fin jurídico, se pone en marcha la administración política, en tanto que cuando lo hace con miras a un fin social, se mueve la administración social. La primera conserva a la sociedad, la segunda preserva a las instituciones de cultura o para fines sociales, que el Estado estableció para crear, promover y proteger.

Como su maestro von Stein, adopta la quíntuple distinción de las ciencias del Estado, que se refieren a las relaciones exteriores, el ejército, la hacienda, la justicia y lo interior, y por lo tanto, la administración que le es inherente a cada cual. Pero, más de modo personal, declara que la administración política comprende a la hacienda, el ejército y lo interior, cuya unidad es el organismo del Estado.

Por otro lado, la administración social se refiere al Estado moderno, en el entendido que "el Estado nacional contemporáneo es además un órgano colectivo, de grandes aspiraciones sociales, hasta de grandes funciones sociales", cuyo origen se encuentran en la ampliación de su esfera de actuación.
No puede decirse que Posada sea un autor original, pues la novedad que aporta: la administración social, no es otra cosa que aquello que Stein definía como administración interna. Su importancia radica en su esfuerzo vigoroso por recuperar el cultivo de la ciencia de la administración, cuando sufría de pasmo en la mayor parte de Europa.

\section{William Willoughby 0 La Ciencia de la Administración Pública en los
Estados Unidos}

Todos estos progresos que se visualizaban en Europa llegaron a los Estados Unidos en la penúltima década del siglo $X \mid X$, cuando Woodrow Wilson publicó su "Estudio de la Administración" en 1887. Casi en paralelo, Frank Goodnow produjo la más célebre de sus obras: Política y Administración, cuya primera edición salió de imprenta en 1900. Después vinieron los trabajos muy importantes de sus discípulos: Leonard White y William Willoughby, quienes respectivamente en 1926 y 1927 produjeron los primeros textos de enseñanza para la administración pública. Willoughby también había escrito otro libro que le dio celebridad: el Goblerno de los Estados Modernos, donde había trazado los grandes aspectos temáticos que estuvieron presentes en la mayor parte de su trabajo académico.

Son muchos e importantes sus méritos, pero destaca en especial ser quien introdujera en los Estados Unidos el concepto de Ciencia de la Administración Pública, ante la tradicional concepción de 
administración pública para referirse doblemente al objeto y al estudio de esta especialidad. Entre los académicos norteamericanos era usual, desde los días posteriores a Wilson, referirse a administración pública como fenómeno y disciplina, al mismo tiempo, en contraste al concepto de Ciencia de la Administración (o Administrativa) que era usual en Europa e lberoamérica. Y su trabajo "The Science of Public Administration" refleja estos problemas epistemológicos en los Estados Unidos (Willoughby, 1937; 39-63).

Willoughby toma posición con referencia al debate, comienza por decir que tratándose de definir como ciencia a la administración, debe comenzarse por conceptuar la noción de ciencia. Primeramente, explica que en administración hay principios generales de aplicación, como en cualquier ciencia, que se refieren a la eficiencia en su operación. Segundo, que estos principios pueden ser determinados, y su significación hecha conocimiento, sólo mediante la aplicación rigurosa del método científico. Hay que recalcar que considera inexcusable que la eficiencia de operación, esté asegurada.

No es menos importante especificar el significado de administración, generalmente referida por medio de una variedad de nociones. Por su parte, dice que la administración entraña a las operaciones totales involucradas en la determinación y puesta en ejecución dentro de una organización, y se extiende al modo como se selecciona el personal, la manera como se usan los recursos materiales y se financian sus operaciones, y el procedimiento por el cual las actividades man- tienen y operan a la empresa de conformidad con su objeto.

Según el autor para entender el significado de administración, es menester hacer algunas distinciones. La primera se refiere a la diferenciación entre administración y legislación, estrictamente hablando, pues ésta generalmente confunde, el establecimiento de la ley como determinación, la regulación de la conducta de los ciudadanos, y las relaciones entre ellos y el Gobierno; con la determinación de cómo el Gobierno, y en particular su rama administrativa, es organizada, cómo labora, cómo se realiza esta labor, cómo se aplica el dinero a ella y cómo se gasta. Ambas tienen igual denominación de "establecimiento de ley", ambas se conocen simplemente como ley o estatuto, pero son diferentes porque la primera tiene un carácter general y permanente, en tanto que la segunda constituye órdenes que se dan a los oficiales públicos a modo de directrices que deben seguir, y la mayor parte de ellas tienen un fin temporal, generalmente anual, y toman con frecuencia la forma de autorización de actos encaminados a objetivos muy precisos, tales como los relativos a las obras públicas. Cuando los cuerpos legislativos estatuyen de la segunda manera, se comportan de modo similar a una junta directiva de una corporación privada, cuando ella imprime la dirección a los negocios de la empresa. En este sentido, los actos de estos cuerpos legislativos son propiamente administrativos, y por extensión son parte de la organización administrativa del Gobierno. Así planteadas las cosas, en los Estados Unidos las directrices primordiales que impulsan el mecanis- 
mos de la administración pública parten del Poder legislativo, no del Poder Ejecutivo.

Otra importante distinción es la referida a los Poderes ejecutivo y administrativo, frecuente y erróneamente confundidos. Así, por ejemplo, los Departamentos del Gobierno nacional son definidos como ejecutivos, aun cuando carecen de autoridad, pues solo son propiamente cuerpos administrativos, salvo con cierta excepción de los Departamentos de Defensa, Marina y Estado, cuyas directrices parten, en varios modos, del Congreso. En su origen, el Poder Ejecutivo no fue identificado como sinónimo de poder administrativo, ni este se incluyó en él, pues este último también quedó repartido con el Poder Legislativo quien ejercita un control.

Una tercera distinción se refiere al trabajo involucrado en la dirección, supervisión y control de las operaciones, y la realización de las operaciones mismas. La primera es la función de dirección, la segunda de administración. Para entender esta distinción, hay que hacer una diferenciación más, a saber, aquella otra que distingue a las actividades institucionales y actividades funcionales. Estas últimas son las que tienen como propósito dirigir la realización del trabajo que es su objeto vital; en tanto que las actividades institucionales son aquellas cuyo objeto es el mantenimiento y operación de la organización misma. Las actividades institucionales son aquella involucradas en políticas determinadas, seguridad y mantenimiento de la planta física, reclutamiento y manejo de personal, así como la contabilidad y la información. Todas estas actividades, que Willoughby juzga esenciales, son distintas sustancialmente a las actividades funcionales no solo por cuanto a clase, sino por el hecho que no son realizadas como un fin en símismo, sino como medios para alcanzar un fin.

Por otra parte, el autor declara que la "Ciencia de la Administración, debidamente construida, tiene poco o nada que hacer sin actividades funcionales". Estas obedecen generalmente a un trabajo técnico muy elevado, pues las tareas que le atañen es de alta complejidad, de modo que el adiestramiento de quienes la desempeñan es parte de la actividad misma. Las actividades funcionales son de dirección, no de administración, que son la materia propiamente de la administración. En tanto las actividades institucionales son de carácter general, las actividades funcionales son de una variedad casi infinita y tienen muy poco en común con aquellas otras, de modo que son aquellas, por su carácter, las que son susceptibles de proyectar al través de principios generales. Por consiguiente, una de las "funciones primarias de la Ciencia de la Administración es determinar cuáles son estos principios y la manera cómo ellos pueden ser aplicados en condiciones variables".

Una vez que Willoughby ha establecido los principios generales que sustentan a la Ciencia de la Administración, procede a identificar los referentes concretos con que trata la disciplina, y que no son otra cosa que las actividades institucionales en si, cuyos problemas clasifica de la siguiente manera:

1. Problemas de administración general

2. Problemas de organización

3. Problemas de material 


\section{Problemas de personal \\ 5. Problemas de procedimiento \\ 6. Problemas de finanzas.}

De este modo, Willoughby sitúa a las actividades funcionales dentro del primer rubro, en tanto que las actividades institucionales permanecen en los demás. Con esta operación del pensamiento, el autor se acerca un poco más que otros de sus compatriotas, con respecto al modo de visualizar a la Ciencia de la Administración sustentada en ambas ramas de actividad, tal como se venía haciendo en Europa desde muchos años atrás.

Tal como lo anunciamos, aquí no hemos tenido más propósito que hacer accesibles a los lectores, a cuatro clásicos de la teoría de la administración pública cuyas contribuciones merecen estar situadas dentro del proceso universal del pensamiento administrativo.

\section{Referencias Bibliográficas}

Bonnin, Charles-Jean Baptiste (1812). Principes d'Administration Publique. $\mathrm{Pa}$ ris, chez Renaudiere Imprimeur-Libraire. Tres volúmenes.

González, Florentino (1840). Elementos de Ciencia Administrativa. Bogotá, Imprenta de J.A. Cualla. Dos volúmenes.

González Saravia, Antonio (1888). La Administración Pública. Guatemala, Establecimiento Tipogrático de la Nación.

Goodnow, Frank (1900). Polltics and Administration. New York, Russell and Russel
Oliván, Alejandro (1843). De la Administra* ción Pública con relación a España. Madrid, Boix Editor.

Posada, Adolfo (Sin techa). La Administración Politica y la Administración Social. Madrid, España Moderna.

Posada de Herrera, José (1978). Lecciones de Administración. Madrid, Instituto Nacional de Administración Pública. La edición original es de 1843. Tres volúmenes.

Rosa, Luis de la (1853). La Administración Pública de México y Medios de Mejorarla. México, sin datos editoriales.

Silvela, Agustín (1839). Estudios Prácticos de Administración. Madrid, Imprenta Nacional.

Stein, Lorenz von (1897). La Scienza della Pubblica Amministrazione. Torino, Unione Tipografico-Editrice.

Vivien, Alejandro (1974), Etudes Administratives. Paris, Editions Cujas. Original publicado en 1845. Dos volúmenes.

White, Leonard (1964). Introducción al Estudio de la Administración Püblica. México, Compañía General de Ediciones. La obra fue publicada originalmente en 1926.

Willoughby, W.F. (1927). Principles of Public Administration. Baltimore. The John Hopkins Press.

"The Science of Public Administration". En John Mathews and James Hart (Eds.). Essays in Political Science. Baltimore, The John Hopkins Press. 1937. pp. 39-63.

Wilson, Woodrow (1887). "The Study of Administration". United States, Polltical Science Quarterly, Vol. 2. 